\title{
The principles of rehabilitation science on the field of regenerative medicine
}

\author{
Ricardo Ferrari, $\mathrm{PhD}, \mathrm{PT}^{1}$ \\ ${ }^{1}$ University of Pittsburgh, School of Medicine - Department of Surgery, Pittsburgh, PA - USA
}

Regenerative medicine is an important field of medicine that combines innovations of tissue engineering, molecular biology, gene therapy, and cellular therapy with the main goal of restoring, repair or replace tissue or stablish normal function following loss due injury, disease, congenital defects, or aging[1]. The common approaches taken to recreate and replace organs and tissues have typically involved a combination of scaffold, cells, and bioactive molecules to stimulate and sustain the body's own self-healing capacity[2].

Adult mammal tissues demonstrate a fantastic regenerative capacity such as bone marrow, liver, and the mucosal epithelium of intestine[3-5]. Similarity, skeletal muscle demonstrates a remarkable ability to regenerate following injury however, this regenerative capacity impairs when a large volume of tissue is lost[6]. Despite the high regeneration capacity of skeletal muscle, the excessive deposition of scar tissue attenuates muscle function and affects the regeneration process after injury[7].

The formation of fibrosis has been demonstrated to compete with myofiber regeneration for the tissue area with inhibition of myofiber function and increase a risk of re-injury. The ability of skeletal muscle to repair itself after injury is largely dependent on the response of muscle stem (satellite) cells (MuSCs). After injury, MuSCs are activated to become myoblasts, which subsequently fuse to form myofibers. However, aging typically culminates in a multitude of compromised MuSC responses. Increasing age typically results in a decreased skeletal muscle regenerative capacity and increased fibrosis after injury, ultimately contributing to a weakness and declines in physical functioning[8].

Skeletal muscle can be physiologically affected by detrimental environment conditions such as long period of immobilization, bed rest, spaceflight, sedentary lifestyle or related to a specific pathology like cancer (cachexia), diabetes, and COVID-19. The skeletal muscle microenvironment, or niche, is an important and potent regulator of MuSC function. The niche is necessary for the maintenance of MuSC quiescence and it provides critical support of tissue 
homeostasis and regenerative capacity [9]. In addition, the niche includes vascular components that are important to deliver homeostatic signals to MuSCs, such as secreted molecules and cellular contacts [10]. However, the biggest challenge for us scientists is to find and develop clinically relevant approaches that might be able to modulate the host microenvironment, maximize functional benefits and improve the regeneration process following cell transplantation.

Researchers on the field of rehabilitation science have started to integrate concepts and techniques used in the clinical setting to enhance healing and restoration of function through tissue regeneration. The integration of regenerative rehabilitation principles with regenerative medicine could promote the goal of developing innovative and effective treatments for skeletal muscle function and repair.

Exercise is a commonly implemented intervention to promote muscle repair in aged muscle and to stimulate the activation of molecular mechanisms critical for functional muscle repair, and emerging evidence indicates that the application of mechanical stimuli increases MuSC activation and proliferation [11]. Upon muscle loading, muscle produces chemical signals that may be essential for MuSC behavior and myogenicity. Importantly, mechanical loading upregulates the gene expression of angiogenic factors, induces growth factor secretion, increases neural recruitment, increases blood flow, and increases leukocyte and monocyte infiltration to the injury area, all of which are critical for adequate regeneration.

There has been intensifying evidence to suggest that muscle contractile activity, by mechanical loading realized with exercise or electrical stimulation (Estim), may also enhance muscle progenitor cell (MPC) regenerative potential $[12,13]$. Indeed, mechanical stimuli has been shown a promising method to improve cells behavior following transplantation. Although, there are many studies demonstrating an increased number, activation, proliferation, and differentiation of MuSCs, the molecular mechanisms underlying these alterations remain poorly understood.

We believe that muscle contractile activity is an effective tool for modulating skeletal muscle regenerative potential, and the expansion of these studies will be important steps towards the development of targeted treatment interventions to prevent muscle wasting and weakness following periods of prolonged immobility, such as after a severe muscle injury, following an intensive care unit stay, or post-operatively. Further research will be necessary to elucidate the mechanisms and pathways to allow us to develop treatment protocols and magnify therapeutic benefits. 
Ferrari R.

\section{References}

1. Perez-Terzic, C. and M.K. Childers, Regenerative rehabilitation: a new future? Am J Phys Med Rehabil, 2014. 93(11 Suppl 3): p. S73-8.

2. Badylak, S.F., Regenerative medicine and developmental biology: the role of the extracellular matrix. Anat Rec B New Anat, 2005. 287(1): p. 36-41.

3. Taub, R., Liver regeneration: from myth to mechanism. Nat Rev Mol Cell Biol, 2004. 5(10): p. 836-47.

4. Jones, M.K., et al., Gastrointestinal mucosal regeneration: role of growth factors. Front Biosci, 1999. 4: p. D303-9.

5. Patt, H.M. and M.A. Maloney, Bone marrow regeneration after local injury: a review. Exp Hematol, 1975. 3(2): p. 135-48.

6. Grogan, B.F., J.R. Hsu, and C. Skeletal Trauma Research, Volumetric muscle loss. J Am Acad Orthop Surg, 2011. 19 Suppl 1: p. S35-7.

7. Kaariainen, M., et al., Relation between myofibers and connective tissue during muscle injury repair. Scand J Med Sci Sports, 2000. 10(6): p. 332-7.

8. Brack, A.S. and T.A. Rando, Intrinsic changes and extrinsic influences of myogenic stem cell function during aging. Stem Cell Rev, 2007. 3(3): p. 226-37.

9. Bursac, N., M. Juhas, and T.A. Rando, Synergizing Engineering and Biology to Treat and Model Skeletal Muscle Injury and Disease. Annu Rev Biomed Eng, 2015. 17: p. 217-42.

10. Watt, F.M. and B.L. Hogan, Out of Eden: stem cells and their niches. Science, 2000. 287(5457): p. 1427-30.

11. Smythe, G.M., et al., Age influences the early events of skeletal muscle regeneration: studies of whole muscle grafts transplanted between young ( 8 weeks) and old (13-21 months) mice. Exp Gerontol, 2008. 43(6): p. 550-62.

12. Ambrosio, F., et al., The synergistic effect of treadmill running on stem-cell transplantation to heal injured skeletal muscle. Tissue Eng Part A, 2010. 16(3): p. 83949.

13. Distefano, G., et al., Neuromuscular electrical stimulation as a method to maximize the beneficial effects of muscle stem cells transplanted into dystrophic skeletal muscle. PLoS One, 2013. 8(3): p. e54922.

\section{Como citar este editorial:}

Ferrari R. The principles of rehabilitation science on the field of regenerative medicine. Rev. Aten. Saúde. 2021; 19(68): 4-6. 